\title{
Weight Excess: The Great Villain in the Development of Hypertension in Adolescents
}

\author{
Erika Maria Gonçalves Campana, ${ }^{1,2}$ Maria Eliane Campos Magalhães, ${ }^{3}$ () Andréa Araujo Brandão ${ }^{1,4}$ \\ Universidade do Estado do Rio de Janeiro (UERJ), ${ }^{1}$ Rio de Janeiro, RJ - Brazil \\ Clínica de Hipertensão Seacor, ${ }^{2}$ Niterói, RJ-Brazil \\ Hospital Universitário Pedro Ernesto da UERJ, ${ }^{3}$ Rio de Janeiro, RJ - Brazil \\ Editorial referring to the article: Hypertension and Different Levels of Body Mass Index and Cardiorespiratory Fitness Amongst Adolescents
}

The relationship between arterial hypertension $(\mathrm{AH})$ and excess weight in young populations is well established. This association involves several conditions including hyperinsulinemia, altered adipokine profile, elevated markers of subclinical inflammation, and inadequate habits, including physical inactivity. ${ }^{1-5}$

In a study $y^{3-5}$ conducted in the city of Rio de Janeiro, Brazil, an increased body mass index in adolescents was correlated with a higher prevalence of elevated blood pressure (BP) and higher risk of developing $\mathrm{AH}$ at young adult age. From a longitudinal perspective, an interesting aspect in the assessment of cardiovascular risk factors is the tracking effect, in which children and adolescents with altered clinical and metabolic parameters (BP, body mass index and cholesterol levels) are likely to maintain an abnormal profile of these variables throughout life and become adults at increased cardiovascular risk. ${ }^{1-5}$ This fact highlights the continuous, unfavorable impact of persistent obesity from childhood to adulthood on cardiovascular risk factors.

The prevalence of physical inactivity at leisure times is very high in Brazilian adolescents (54.3\%. 95\%CI 53.4-55.2), especially among girls. One fourth of the adolescents (26.5\%; 95\%CI 25.8-27.3) reported to be physically inactive at leisure times (girls $39.8 \%$, 95\%CI $38.8-40.9$ vs. boys $13.4 \%$, 95\%CI $12.4-14,4) .{ }^{6}$ Although these prevalence data have indicated a

\section{Keywords}

Hypertension; Adolescents; Sedentarism; Physical Activity; Exercise; Quality of Life; Risk Factors; Obesity. correlation between excess weight, physical inactivity, and hypertension in young individuals, there are still important evidence gaps regarding this association. ${ }^{1-5}$

In the current issue of the International Journal of Cardiovascular Sciences, Bertollo et al., ${ }^{1}$ explored the interrelationship between obesity, cardiorespiratory fitness, and hypertension in 860 adolescents. The authors found a prevalence of $22 \%$ of elevated BP in overweight/ obese individuals with low cardiorespiratory fitness, and a prevalence of $15 \%$ in those overweight with adequate cardiorespiratory fitness. In underweight and normal weight adolescents, the frequency of hypertension was relatively lower, and independent of the cardiorespiratory fitness level $(14.6 \%$ and $15.7 \%$ in those with adequate and low cardiorespiratory fitness levels, respectively). ${ }^{6}$ Thus, cardiorespiratory fitness had no effect on the positive correlation between excess weight and the presence of hypertension. This study adds information about the relationship between BP, excess weight, and physical fitness in adolescents, which is of particular importance considering the scarcity of studies on this subject with individuals in this age range in Brazil. However, the cross-sectional nature of the study precludes establishing a causal relationship between these variables as well as analyzing the impact of a higher cardiorespiratory fitness in a longer-term perspective.

Likewise, in a cross-sectional study, Briggs et al., ${ }^{7}$ evaluated the relationship between cardiorespiratory fitness, prevalence of cardiovascular risk factors, and risk of metabolic syndrome in 183 young obese individuals. Of the study individuals, 30\% had metabolic syndrome, but no differences were

\section{Mailing Address: Erika Campana}

Hospital Universitário Pedro Ernesto. Departamento Cardiopulmonar

Boulevard 28 de Setembro, 77. Postal Code: 20551-030, Vila Isabel, Rio de Janeiro, RJ - Brazil.

E-mail: campanaemg@gmail.com 
observed in the prevalence of cardiovascular risk factors or metabolic syndrome between individuals with higher and lower cardiovascular fitness.

Martínez-Vizcaíno et al., ${ }^{8}$ evaluated the effects of a physical activity program on indicators of obesity, cardiorespiratory fitness, and BP in children. This was a crossover randomized clinical trial including 1,434 children (4-7 years old). The intervention consisted of three 60-minute sessions per week on weekdays between October 2013 and May 2014. Despite improvements in cardiorespiratory fitness seen in both girls $(p<0.001)$ and boys $(p<0.001)$, there were no significant improvements in overweight/obesity with the intervention compared with the control group in both sexes. Also, the intervention did not alter other adiposity indicators or BP parameters.

Therefore, studies with individuals in this age range have suggested that body composition is the key point in the relationship between $\mathrm{BP}$ and physical fitness and is sufficient grounds for promoting the regular practice of physical activity. However, excess weight seems to play a fundamental role in the development of hypertension and of an abnormal metabolic profile in children and adolescents. Long-term, longitudinal studies may help to understand the impact of regular physical activity and higher cardiorespiratory fitness in early stages of life on BP and other cardiovascular risk factors.

In a modern view of $\mathrm{AH}$ and cardiovascular risk, vascular injury and an accelerated vascular aging are seen as indicators of increased risk of cardiovascular morbidity and mortality since early age. ${ }^{2}$

The study conducted in Rio de Janeiro was one of the pioneers in the evaluation of the relationship between arterial stiffness and excess weight in the young. The study participants $(\mathrm{n}=96)$ were stratified into three groups according to the pulse wave velocity tertile for each sex. The group in the highest tertile showed higher mean values for body mass index, BP, systolic and diastolic BP, insulin, and HOMA-IR, lower adiponectin levels, and higher prevalence of diabetes mellitus, glucose intolerance and hyperinsulinemia. Pulse wave velocity showed a significant positive correlation with body mass index, systolic and diastolic BP, mean BP, pulse pressure and low-density lipoprotein cholesterol levels, and a significant negative correlation with high-density lipoprotein-cholesterol and adiponectin. In the multiple regression model, after adjustment for HDL-cholesterol, LDL-cholesterol and adiponectin by sex, age, body mass index and mean BP, only male sex and mean BP remained significantly correlated with pulse wave velocity. ${ }^{9}$

The association between body composition, cardiorespiratory fitness and arterial stiffness in young Swedish adults was investigated by Fernberg et al., ${ }^{10}$ The authors found a negative correlation of body mass index/cardiorespiratory fitness with pulse wave velocity. Young adults with obesity and low cardiorespiratory fitness had significantly higher pulse wave velocity than non-obese young adults with medium or high cardiorespiratory fitness. In the multiple regression analysis, cardiorespiratory fitness had a stronger effect on arterial stiffness as compared with body mass index.

This association between low cardiorespiratory fitness and arterial stiffness was also observed by Haapala et al., ${ }^{11}$ in a study with children with chronic diseases. In their study, low cardiorespiratory fitness and increased waist circumference were associated with increased arterial stiffness in children and adolescents with chronic diseases and physical disabilities.

The role of physical exercise in vascular health is still controversial. Intense resistance training, without additional aerobic exercises, can increase pulse wave velocity in healthy young individuals. However, aerobic exercise seems to be a strategy capable of maintaining or restoring healthy arterial aging. This favorable effect of aerobic exercise on vascular health seems to occur regardless of its effect on BP; it involves anti-inflammatory and anti-proliferative effects and influences vasodilation of small and mediumsized vessels that directly affect wave reflection, and consequently augmentation index and central systolic pressure. $^{12}$

The meta-analysis by Cote et al., ${ }^{13}$ compared arterial stiffness measures of obese children and adolescents with those of a control group with normal body mass index. The authors demonstrated a significant effect of obesity on pulse wave velocity, indicating greater arterial stiffness in children and adolescents with obesity than controls.

Therefore, overweight seems to be the key element associated with different clinical scenarios including elevated BP, inadequate habits, altered metabolic profile, cardiovascular risk factors and subclinical vascular abnormalities. Maintenance of an adequate body weight should be one of the pillars of primary prevention strategies in young populations. 


\section{References}

1. Bertollo C, Barbian CD, Schneiders LB, Silveira JF, Vogt BD Mello ED, et al. Hypertension and Different Levels of Body Mass Index and Cardiorespiratory Fitness Amongst Adolescents. Int J Cardiovasc Sci. 2021; 34(6):610-616. DOI: https://doi.org/10.36660/ ijcs. 20200038

2. Barroso WKS, Rodrigues CIS, Bortolotto LA, Mota-Gomes MA, Brandão AA, Feitosa ADM, et al. Brazilian Guidelines of Hypertension - 2020. Arq Bras Cardiol. 2021 Mar;116(3):516-658. doi: 10.36660/abc.20201238.

3. Campana EM, Brandão AA, Pozzan R, Magalhães ME, Fonseca FL, Pizzi $\mathrm{OL}$, et al. Blood pressure in adolescence, adipokines and inflammation in young adults. The rio de janeiro study. Arq Bras Cardiol. 2014 Jan;102(1):60-9. doi: 10.5935/abc.20130226.

4. Campana EMG, Brandão AA, Pozzan R, França MF, Fonseca FL, Pizzi $\mathrm{OL}$, et al. Blood pressure in young individuals as a cardiovascular risk marker. The Rio de Janeiro study. Arq Bras Cardiol. 2009 Dec;93(6):60815, 657-65. doi: 10.1590/s0066-782×2009001200016.

5. Brandão AA. Obesity trebles in Brazilian schoolchildren over a 30-year period. Eur Heart J. 2017;38(8):538. doi:10.1093/eurheartj/ehx054

6. Cureau FV, da Silva TL, Bloch KV, Fujimori E, Belfort DR, de Carvalho $\mathrm{KM}$, et al. ERICA: leisure-time physical inactivity in Brazilian adolescents. Rev Saude Publica. 2016 Feb;50 Suppl 1(Suppl 1):4S. doi: 10.1590/S015188787.2016050006683

7. Briggs MS, Spees C, Bout-Tabaku S, Taylor CA, Eneli I, Schmitt LC. Cardiovascular risk and metabolic syndrome in obese youth enrolled in a multidisciplinary medical weight management program: implications of musculoskeletal pain, cardiorespiratory fitness, and health-related quality of life. Metab Syndr Relat Disord. 2015 Apr;13(3):102-9. doi: 10.1089/met.2014.0107.

8. Martínez-Vizcaíno V, Pozuelo-Carrascosa DP, García-Prieto JC, CaveroRedondo I, Solera-Martínez M, Garrido-Miguel M, Díez-Fernández A, Ruiz-Hermosa A, Sánchez-López M. Effectiveness of a school-based physical activity intervention on adiposity, fitness and blood pressure: MOVI-KIDS study. Br J Sports Med. 2020 Mar;54(5):279-85. doi: 10.1136/ bjsports-2018-099655.

9. Pizzi OL, Brandão AA, Pozzan R, Magalhães ME, Campana EM, Fonseca $\mathrm{FL}$,et al. Pulse wave velocity, blood pressure and adipocytokines in young adults: the Rio de Janeiro study. Arq Bras Cardiol. 2013 Jan;100(1):60-6. doi: $10.1590 /$ s0066-782×2012005000116.

10. Fernberg U, Fernström M, Hurtig-Wennlöf A. Arterial stiffness is associated to cardiorespiratory fitness and body mass index in young Swedish adults: The Lifestyle, Biomarkers, and Atherosclerosis study. Eur J Prev Cardiol. 2017 Nov;24(17):1809-18. doi: 10.1177/2047487317720796.

11. Haapala EA, Lankhorst K, de Groot J, Zwinkels M, Verschuren O, Wittink $\mathrm{H}$, et al. HAYS study group. The associations of cardiorespiratory fitness, adiposity and sports participation with arterial stiffness in youth with chronic diseases or physical disabilities. Eur J Prev Cardiol. 2017 Jul;24(10):1102-11. doi: 10.1177/2047487317702792.

12. Nowak KL, Rossman MJ, Chonchol M, Seals DR. Strategies for achieving healthy vascular aging. Hypertension. 2018; 71(3): 389-402.

13. Cote AT, Phillips AA, Harris KC, Sandor GG, Panagiotopoulos C, Devlin AM. Obesity and arterial stiffness in children: systematic review and meta-analysis. Arterioscler Thromb Vasc Biol. 2015 Apr;35(4):1038-44. doi: 10.1161/ATVBAHA.114.305062. 